\title{
Large-Area Photogrammetry Based Testing of Wind Turbine Blades
}

\author{
Peyman Poozesh $^{* a}$, Javad Baqersad ${ }^{\mathrm{b}}$, Christopher Niezrecki $^{\mathrm{a}}$, Peter Avitabile ${ }^{\mathrm{a}}$ \\ Eric Harvey ${ }^{\mathrm{c}}$, Rahul Yarala ${ }^{\mathrm{c}}$
}

An optically based sensing system that can measure the displacement and strain over essentially the entire area of a utility-scale blade leads to a measurement system that can significantly reduce the time and cost associated with traditional instrumentation. This paper evaluates the performance of conventional three dimensional digital image correlation (3D DIC) and three dimensional point tracking (3DPT) approaches over the surface of wind turbine blades and proposes a multi-camera measurement system using dynamic spatial data stitching. The potential advantages for the proposed approach include: (1) full-field measurement distributed over a very large area, (2) the elimination of timeconsuming wiring and expensive sensors, and (3) the need for large-channel data acquisition systems. There are several challenges associated with extending the capability of a standard 3D DIC system to measure entire surface of utility scale blades to extract distributed strain, deflection, and modal parameters. This paper only tries to address some of the difficulties including: (1) assessing the accuracy of the 3D DIC system to measure full-field distributed strain and displacement over the large area, (2) understanding the geometrical constraints associated with a wind turbine testing facility (e.g. lighting, working distance, and speckle pattern size), (3) evaluating the performance of the dynamic stitching method to combine two different fields of view by extracting modal parameters from aligned point clouds, and (4) determining the feasibility of employing an output-only system identification to estimate modal parameters of a utility scale wind turbine blade from optically measured data. Within the current work, the results of an optical measurement (one stereo-vision system) performed on a large area over a 50-meter utility-scale blade subjected to quasistatic and cyclic loading are presented. The blade certification and testing is typically performed using International Electro-Technical Commission standard (IEC 61400-23). For static tests, the blade is pulled in either flap-wise or edgewise directions to measure deflection or distributed strain at a few limited locations of a large-sized blade. Additionally, the paper explores the error associated with using a multi-camera system (two stereo-vision systems) in measuring 3D displacement and extracting structural dynamic parameters on a mock set up emulating a utility-scale wind turbine blade. The results obtained in this paper reveal that the multi-camera measurement system has the potential to identify the dynamic characteristics of a very large structure.

Keywords: Point tracking, full-field strain, wind turbine blade, structural health monitoring, operational modal analysis.

\section{INTRODUCTION}

As wind turbine blade manufactures are pushing the envelope of design and moving toward larger scale blades, the need for effective non-destructive evaluation (NDE) and a better understanding of blade dynamics increases. The current state of practice to inspect wind turbine blades relies on traditional sensors (e.g. strain gages, string potentiometers, and accelerometers) that provide information at a number of discrete points. [1-4]. However, the complex dynamic response of these sizable blades cannot be quickly or easily measured using a limited set of mounted accelerometers. Placing transducers on a utility-scale wind turbine blade is labor intensive, requires a multitude of sensors and hardware, and can also introduce electrical noise to the measured signal due to the extensive wiring. A typical 50m utility-scale blade 
requires $\sim 200$ gages (costing $\$ 35 \mathrm{k}-\$ 50 \mathrm{k}$ ), takes $\sim 3$ weeks to set up. Performing accurate modal tests requires numerous, low frequency, and high sensitivity accelerometers that can be very expensive (e.g. $\$ 300$ to $\$ 1400$ per sensor) along with the associated signal conditioners. If a non-contact measurement system was available that could measure full-field deflections and distributed strain, it would be possible to streamline the blade testing process by eliminating instrumentation and sensors and saving set up time (e.g. typically 3-4 technicians working for 2 days). Techniques such as using a scanning laser Doppler vibrometer (SLDV) are limited to sequential measurement (i.e. one point at a time) and to obtain information at many points the laser measurements must be tested in series [5-7]. Additionally, measuring very large displacements as found on wind turbine blades using a SLDV is not possible because the large motions of the structure cause the targeted laser position to move on the structure. However, digital image correlation (DIC) can measure the full-field response of the structure and is not sensitive to large deformations. Stereo-photogrammetry in the context of this paper uses 3D DIC and 3DPT techniques. These approaches provide the ability to collect 3D dynamic measurements at many more points on a structure compared to conventional testing using strain gages, accelerometers, or displacement sensors. However, for large scale structures within a test facility or even outdoors, the use of stereophotogrammetry to measure large dimensions may be limited by the camera separation or the required working distance to capture the field of view of interest. Owing to a stereo-vision system's limited field of view, large-scale test articles and structures with complex curvatures need to be measured from many different viewing directions. The knowledge gained by developing a multi-camera system will lead to a measurement system that can significantly reduce the time and cost associated with strain gage instrumentation; thereby, reducing the time required for blade certification and levelized cost of energy (LCOE). The current lack of multi-camera stereo-photogrammetry measurement to accurately assess the operating deflection shapes of large structures with complex geometry has not been sufficiently addressed by the scientific community and provides the motivation for this work.

This paper extends the understanding of 3D DIC measurement capabilities by evaluating the performance of a multicamera 3D DIC system in which data obtained with conventional DIC systems are stitched together in a universal coordinate system. The primary objectives are to quantify the performance of conventional 3D DIC by conducting a fullfield experiment on a large area (i.e. wind turbine blade) and evaluate the performance of a multi-camera DIC system in extracting the modal parameters on a test structure. A review of the previous efforts in these areas is summarized in the following sections. An example of the types of measurements that can be acquired using DIC over a large area is shown for a utility-scale wind turbine blade in Section 2. Following, how multiple fields of view are stitched together and an analysis of the associated errors of the approach are presented for a wind turbine blade mock up.

\subsection{Prior Work Related to Photogrammetry}

Full-field measurement using non-contact techniques to extract desired dynamic characteristics of structures led researchers to develop optical measurement systems. Pattern interferometry, laser Doppler interferometry, and photogrammetry are three types of optical methods [8]. The interferometry techniques such as ESPI and DSS, developed prior to DIC system, have been employed to measure full-field response of structures subjected to dynamic and static loading. The interferometry techniques use an interference fringe pattern created by the superposition of two coherent light patterns and measure the displacement between these patterns.

Van der Auweraer et al. [9] used pulsed-laser holography, or electronic speckle pattern interferometry (ESPI), to perform vibration testing. Zanarini [10] used ESPI for a broad frequency band vibration measurement and compared the performance of ESPI with SLDV and DIC [5,6]. In other attempts ESPI technique was employed to obtain full-field vibration measurements in the fatigue behavior assessment of mechanical components by means of spectral approaches [11-13]. A laser Doppler vibrometer (LDV) scans each point sequentially; therefore compared to 3D DIC and ESPI, a LDV may not have the consistency in the measurement as the physical excitation or the structure changes over time during a measurement.

Using either a LDV or interferometry techniques is not well suited to making measurements on utility-scale wind turbine blades because of the very large deformations these structures exhibit during fatigue testing. Additionally, both of these approaches are susceptible to noise measurement, which can be problematic when testing in a field setting or location with ground vibrations. Stereo-photogrammetry is better suited for making measurements at lower frequencies when the structures' displacements are large [14]. A fair amount of research has been conducted to improve stereophotogrammetry techniques, three-dimensional digital image correlation (3D DIC) and three-dimensional point tracking (3DPT) as an alternative to conventional point-wise and contacting measurement sensors. Both 3D DIC and 3DPT are non-contact image based techniques that rely on a series of images taken with two digital cameras to track the motion of 
an object in three dimensions. Although 3DPT is not technically a full-field measurement technique because it measures motion at discrete points, for all practical purposes it can be considered so because it is relatively easy and inexpensive to mount numerous optical targets to fully cover the structure of interest throughout the field of view (FOV). The DIC approach is an optical measurement technique that includes image processing and numerical computation to measure three-dimensional shape and deformation. The geometry and deformation is measured by recording a speckled pattern on the surface of the structure (typically black and white) and monitoring how the pattern deforms when multiple images from the sensor pair are captured [15-17]. Likewise, 3DPT can be used to measure displacement at discrete points by mounting optical targets or fiducial markers to the structure. DIC and 3DPT have matured over the last two decades and have been primarily applied to the field of experimental solid mechanics. However, more recently researchers have begun to exploit the optically based approach for measuring vibration and high-speed transient phenomena.

The stereo-photogrammetric measurement technique has been employed to measure large utility-scale wind turbine blades. Ozbeket al. [18-21] applied the 3DPT to a 2.5-MW Nordex N80 wind turbine with an 80-meter tower height and rotor diameter. They also showed that modal parameters of the rotating utility-scale wind turbine blade can be identified using stereo-photogrammetric measurement. Paulsen et al. also utilized 3DPT to measure the operating deflection shapes of a 41-meter diameter wind turbine blade [22]. In another effort, the 3D DIC method was used to determine surface geometry, deformation, and strain distribution over a large area on a 50-meter utility-scale blade subjected to quasi-static and cyclic loadings [23]. However, it was constrained to measure only a portion of the utility scale wind turbine blade.

\subsection{Prior Work Related to Spatial Stitching}

To extend DIC stereo-photogrammetry capabilities, some researchers have been working on a multi-camera DIC system ( $\mathrm{N}$ cameras, e.g. $\mathrm{N}=4,6,8, \ldots$ ) in which point clouds obtained with several individual standard DIC systems are stitched together in a global coordinate system[24-26]. In order to obtain full-field displacement and strain on a utility-scale wind turbine blade using a single camera pair, the blade's entire surface must be visible by both cameras. However, due to a sensor's field of view limitation, spatial constraints of the testing facility and optical occlusion effects or complex curvature, large-scale wind turbine blades need to be measured from many different viewing positions or directions as seen in Figure 1. Three different approaches have been suggested to stitch the different fields of view provided by multiple DIC measurements [27]. The first approach fixes the sensors and moves test object on a mechanical stage. This method is generally not feasible for large-scale inspection of structures subjected to static or dynamic mechanical loading. The second method involves moving the stereo vision sensors around the fixed test object. LeBlanc et al.[28] used a portable stereo DIC setup system to measure the strain and displacement over the entire surface of a nine-meter TPI Composites CX-100 blade with and without an applied load. The entire blade was divided into 16 different fields of view. These fields of view were captured with a single stereo DIC system by repositioning the setup. To obtain continuous data across the entire blade surface, a technique to combine multiple fields of view was developed. Although this method was successful in providing a full-field strain and displacement measurement over the entire 9-meter blade, it is limited to static measurements and is not appropriate for transient or cyclic loading. The third approach is to use a multi-camera stereo DIC setup to obtain full-field displacements and strain fields [25]. The setup is composed of multiple standard stereo DIC systems, each of which is calibrated independently. This procedure can be applied when the images taken by each individual stereo DIC system are overlapped by a certain amount to ensure two or more images can be stitched [29]. Figure 2 depicts the schematic plot of a multi-camera DIC system that Wang et al. [25] developed to cover the blind spots on the objects with complex curvatures. Chen et al. performed full-field 3D measurement on a panel using a multi-camera DIC system. Multiple cameras are calibrated as a single camera system. The measured data by using multiple pairs of cameras is mapped into a universal coordinate system based on the calibration information. A subset based matching technique looks through all the captured images to find the best match for a specific single subset. If the subset can only be found in one pair of cameras, the data obtained from that pair of cameras will be used to do 3D reconstruction. Their multi-camera system depends on the symmetry and stability of the setup. All cameras in the setup must be fixed rigidly during the test [28]. 


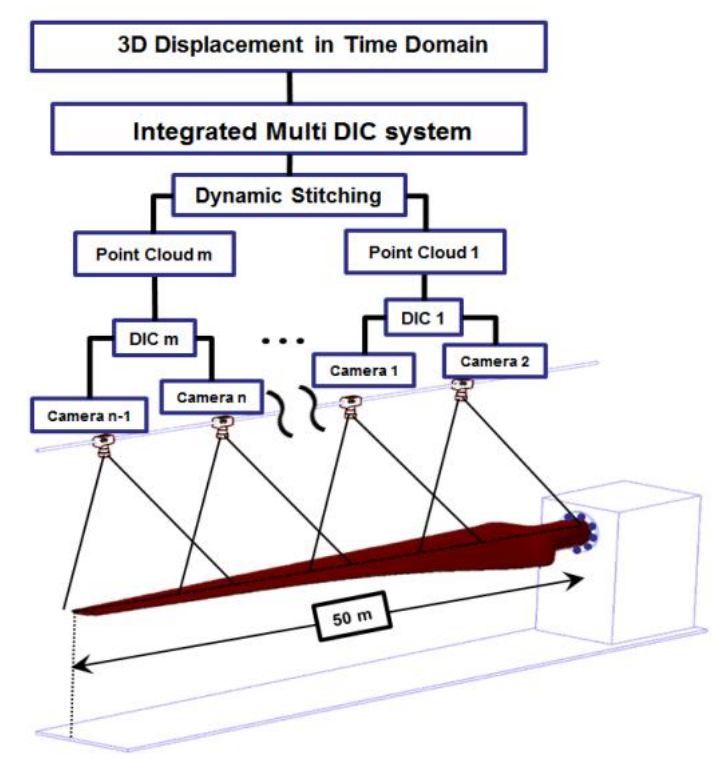

Figure 1. A schematic of a proposed multi-camera DIC system consisting of multiple independent standard stereo-vision systems that can be used for full-field DIC measurement on utility scale wind turbine blades.

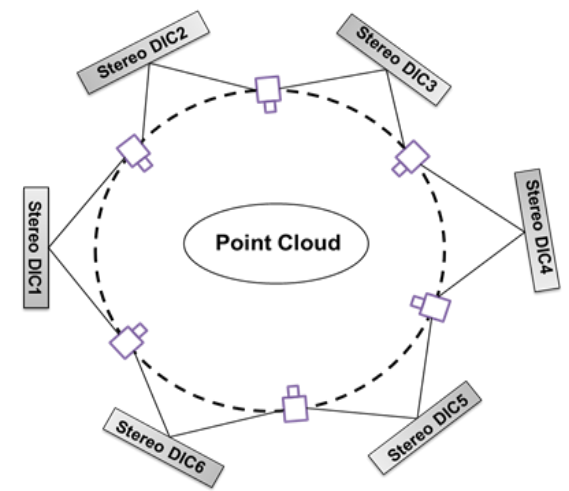

Figure 2. A schematic plot of the proposed multi-camera DIC system to perform measurement on an object with complex curvature [25].

In the current work, the potential of 3D DIC method in measuring strain and displacement over a very large area on a 50meter utility-scale blade subjected to quasi-static and cyclic loading is evaluated. Identifying the unique constraints associated with making 3D DIC measurement within the blade test facility will help to develop an optical sensing technique that can measure full-field 3D displacement and strain over essentially the entire utility scale blade. Furthermore, a multi-camera system (composed of two independently calibrated stereo-vision systems), is used to obtain the displacement fields and operating shapes of a Southwest Wind power Skystream 4.7 wind turbine blade. The wind turbine blade containing a handful of mounted optical targets is excited using an unknown impact force and a standard 3DPT system is used to measure the 3D displacement on a limited area of the blade. Following the data collection, the displacement fields are stitched together to reconstruct the global 3D displacement field as a point cloud. It is important to mention that the same hardware (i.e. data acquisition system, cameras, computer, and calibration) are used to make either DIC or 3DPT measurements. Stitching of the obtained geometry and displacement fields is performed by finding transformation matrices that relate corresponding points in the overlap areas. The rotation and translation matrices are computed by using point cloud registration techniques such as Singular Value Decomposition (SVD) or Iterative Closets Point (ICP). An appropriate stitching process expresses the resulted fields in the coordinate system of the master camera. Within this paper SVD based image registration is used to align two different point clouds measured by different stereovision systems.

This work evaluates the capability of a single stereo-vision system and multi-camera 3D DIC in extracting dynamic characteristics of large structures. The multi-camera 3D DIC measurement approach proposed in this paper will enable 
engineers to monitor the full-field strain and displacement of very large areas that require several cameras spanning multiple fields of view. Although not all the issues related to implementing a multi-camera system within the wind turbine testing facility are completely addressed in this work, the current paper paves the way for developing an optical measurement system that can be used to perform full-sized structural evaluation of a utility scale wind turbine blade.

\section{FULL SURFACE CHARACTERIZATION OF UTILITY SCALE WIND TURBINE BLADE USING ONE STEREO-VISION SYSTEM}

To implement a 3D DIC test on a large area, the following consecutive steps were taken: 1) prepare the test structure by applying a high contrast white and black stochastic pattern to its surface over the area of interest;2) record images of the specimen surface before and after loading using a calibrated sensor pair; and 3) process the acquired images using a computer program to obtain the desired displacement and strain information. The experiment to evaluate the feasibility of using the 3D DIC technique over a very large area was executed on AMI blade (Advanced Manufacturing Initiative) at the Wind Technology Testing Center (WTTC). The composite utility-scale blade was mounted to a large concrete anchor which is used to cantilever the blade for both static and fatigue loading. In the static test the blade is displaced in flap-wise or edgewise to measure blade deflection and strains. The fatigue test is performed using hydraulic actuators that oscillate the blade in its first natural frequency. Optical measurements were performed on the low pressure side of the blade located near the root of the blade. Although the root section has a very small modal contribution (e.g. little displacement), the location was chosen primarily to demonstrate the feasibility of the measurement approach on a utilityscale turbine, for logistical convenience, and because the root section has high strains, is susceptible to failure, and is an area of interest to blade manufacturers. The measurement area on the blade and experimental test setup along with imaging devices are shown in Figure 3. Before performing 3D DIC measurements, a pattern check and noise floor assessment was carried out. A measurement was executed on the speckle patterned area when the blade was stationary and the blade was under its own weight. Using DIC software (Aramis by GOM), a 3D geometry of a portion of the blade that contains speckle pattern, near the root of the blade, was generated. The measured 3D geometry can be used to update the CAD model of the blade and 3D shape deviation calculations [30] (see Figure 4). As shown in Figure 4, the extracted 3D geometry reveals the strain-gage wiring on the blade. The first set of images, recorded before applying loads to the blade, are used for noise floor measurements and to check the quality of the speckle pattern on the blade.
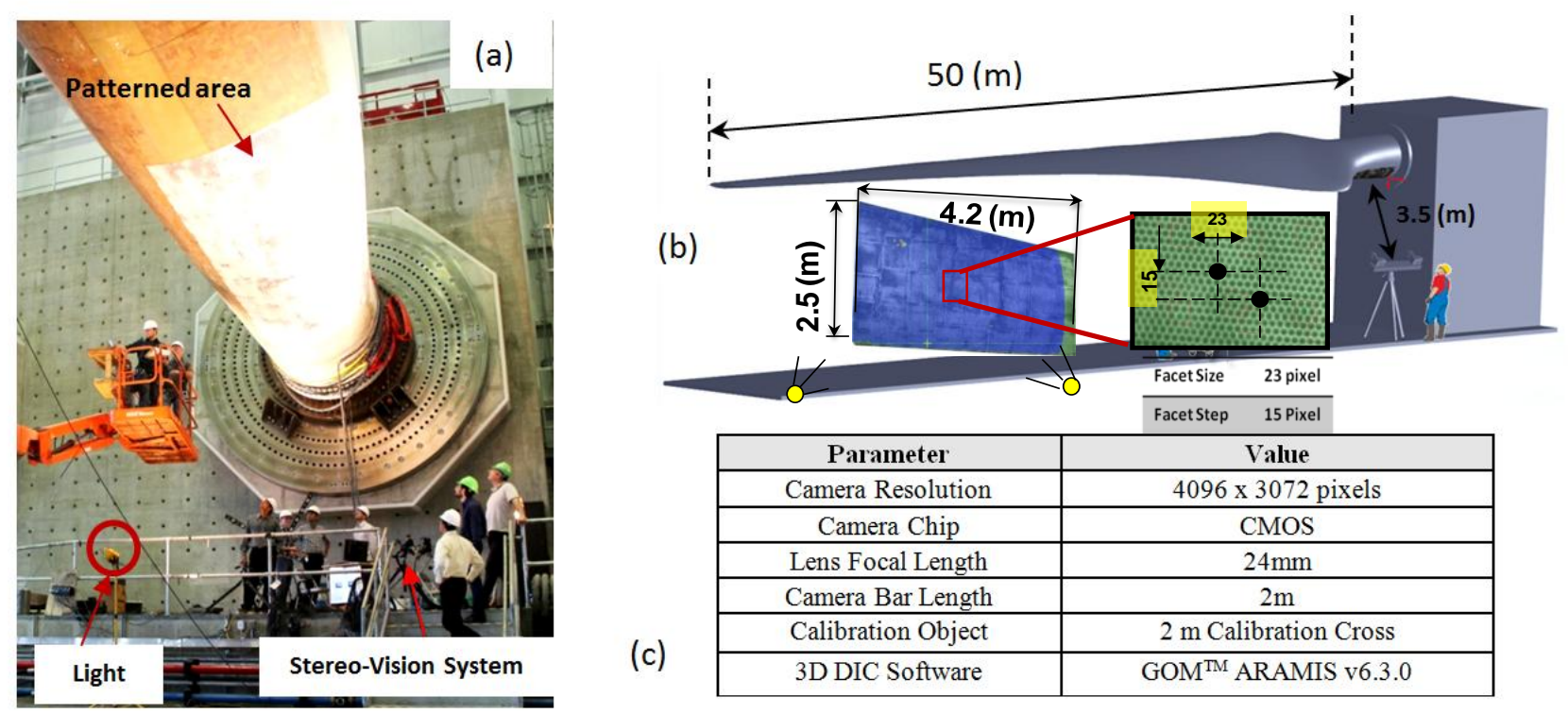

Figure 3. Description of test setup: (a) photograph of the test set up at the WTTC showing the patterned area of the AMI (Advanced Manufacturing Initiative) blade test set up; (b) a schematic of utility scale blade and portion of the blade area with speckle pattern and facet size and step used to process images; (c) the specifications of the optical devices used for capturing the images.

Given the size of the measurement area, approximately 4 meters long and 3 meters wide, an extended calibration method was employed to calibrate the DIC system. A two-meter calibration crossbar was used to calibrate a field of view up to 
approximately 4 meters wide, referred to as an extended calibration. The normal calibration creates a calibrated measuring volume that is approximately the same width as the calibration test object (see [37] for more details). To prepare the area for strain and displacement monitoring, a black and white speckled pattern was painted on the blade surface. A proper pattern is one that has high contrast (large gray-scale variation) with appropriate size dots ( $\sim 6$ pixel in diameter). Photogrammetric targets were also applied to the patterned area and serve two purposes. First, the targets can be used to define a coordinate system for the strain and displacement analysis. Second, the targets can be used as simple extensometers. Recorded images with a stereo-vision system were used to determine surface geometry, strain, and displacement in three dimensions on the part of the blade surface that previously had a speckle pattern applied to it. Besides using two additional lights (1000 watt), the ambient light in the test facility was sufficient to perform optical measurements during static and fatigue tests. Image contrast is the most important lightning feature that can influence the performance of the DIC test.

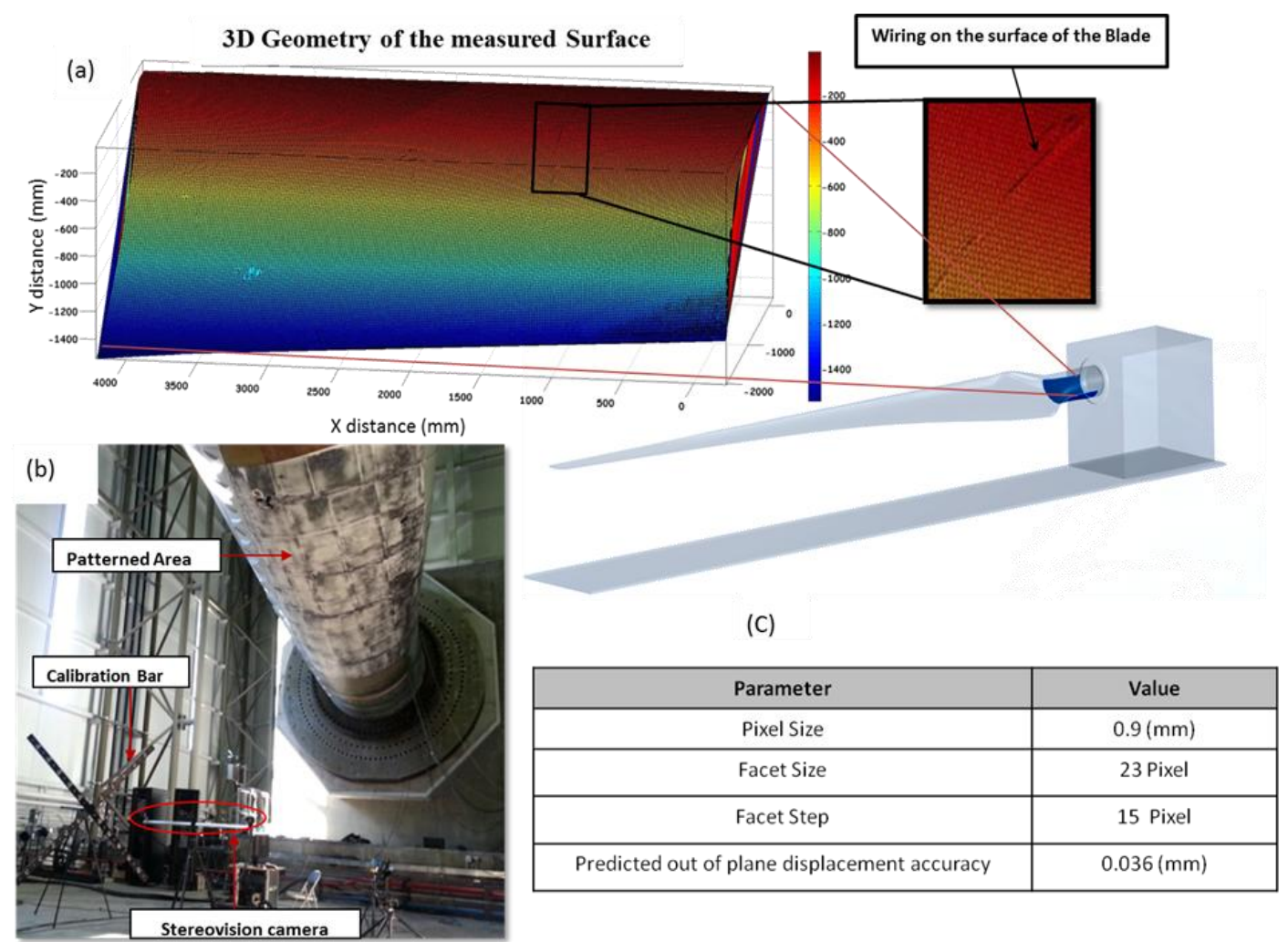

Figure 4. Test set up and the geometry of the test object: (a) an example of the full-field surface geometry over a portion of the utility scale blade area; (b) photograph of the test set up at the WTTC showing the patterned area; (c) DIC parameters used to compute displacement.

\subsection{Quasi-static test}

Quasi-static tests of the wind turbine blades are required for certification purposes. For this test, the wind turbine blade is statically loaded in the flap-wise direction. This type of testing is carried out to validate finite element models and to assess the blades ability to withstand extreme loads. Static testing on the AMI blade at the WTTC was performed with a series of computer controlled servo-hydraulic winches. Figure 5 shows a schematic of AMI blade subjected to the incremental static loading. The blade was incrementally exposed to quasi static loads till the maximum design load was verified; the static loads were induced in five steps. The loading location and the measurement area are shown in Figure 5.

The static load testing was performed while the patterned area was viewed by a pair of high resolution, digital cameras. The images captured by the stereo-vision camera system were processed using the DIC technique and the deformation of the measurement area was computed. The full-field displacement of the area of interest is shown in Figure 6 . The out of plane displacement uncertainty was predicted to be 0.04 pixels ( $\sim 36$ micron); however the actual uncertainty of the 
measured displacement data was shown to be $+/-65$ micron. The measurement uncertainty was determined by comparing the displacement over the entire field of view using 10 image pairs that are sampled while the blade was assumed to be stationary. The variation of the calculated displacement provides the noise floor of the measurement. The highest variation of the displacement or strain, while the blade is subject to neither static nor dynamic force yields the uncertainty of measurement. The displacements of the three discrete points (Point A, B, and C) on the measurement surface are compared in Figure 6. These points represent the approximate location of mechanical strain gages mounted on the utility-scale blade. Figure 6 also shows the curvature of the blade, as function of section length. The selected section line in Figure 6 is located on the spar cap and aligned along the length of the blade.
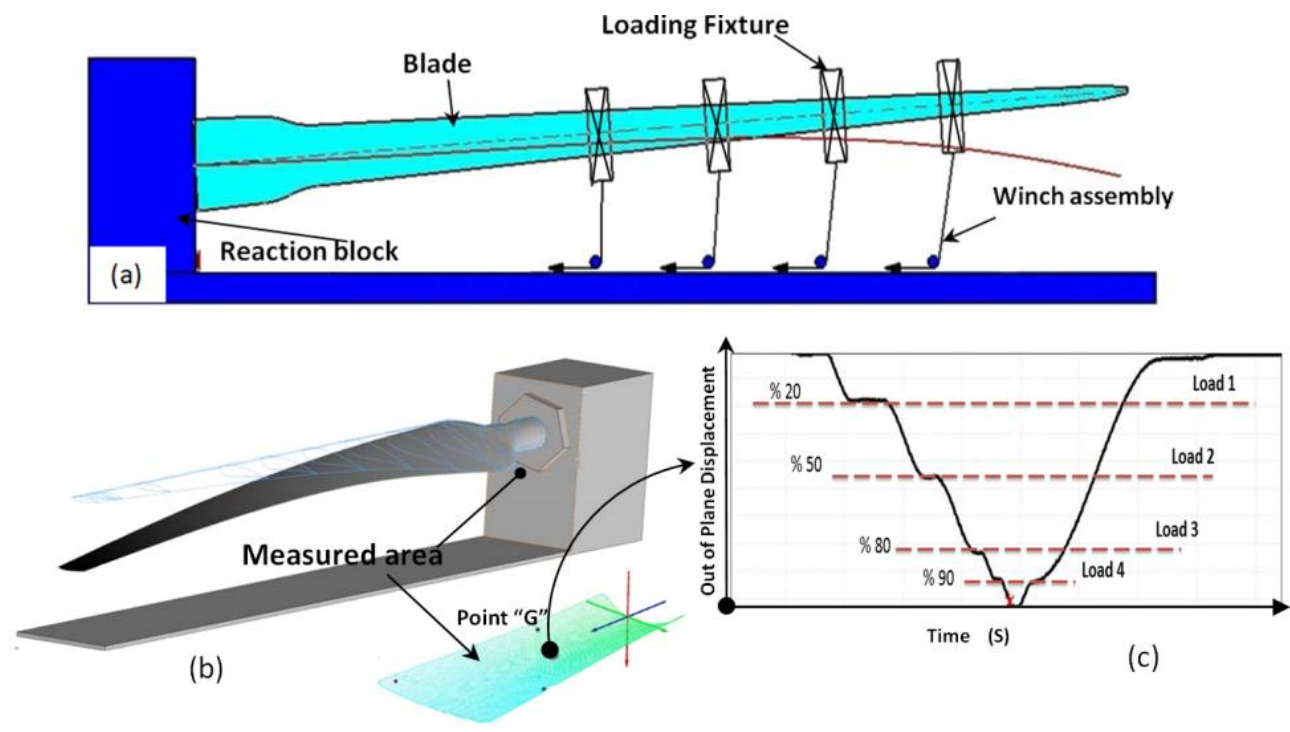

Figure 5. Planform view of the AMI wind Turbine blade indicating measurement and loading sections: (a) location of the loading sections; (b) schematic of the blade after being subjected to static loading and measurement area; (C) the measured displacement of the blade at a single location using DIC for various loading levels.
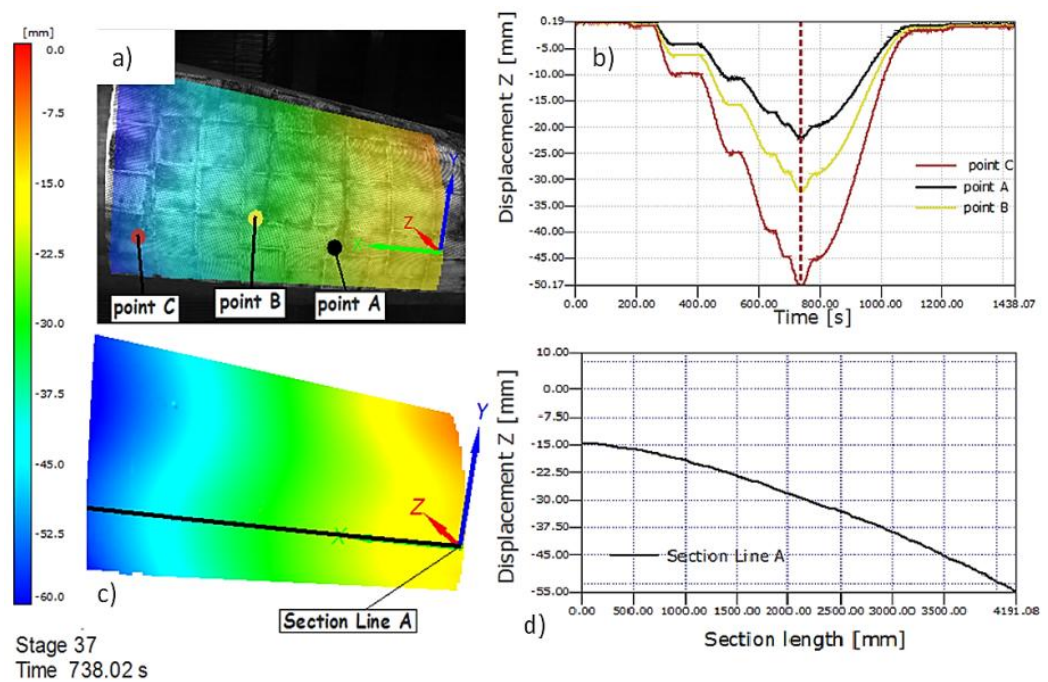

Figure 6. Measurement displaying curvature of blade along the low-pressure surface: (a) overlay of blade image and spatial displacement contour; (b) a comparison of out of plane displacement values at various locations; (c) spatial displacement contour near the blade root (on the right); (d) displacement section line along the length of the blade (root shown on left). 
Measuring the strain distribution of the load carrying spars that runs the length of the blade is important in understanding the blade behavior. Having employed the 3D DIC system, the captured images from the 3D patterned area before and after deformation were used to map the strain distribution. The full-field strain contour over the area of the interest is shown in Figure 7. The results obtained in Figure 7 provide important information regarding the strain distribution on the surface of the blade. Strain values of three different points on the spars are compared in Figure 7 for increasing, then decreasing loads over time. Furthermore, the strain variation along the longitudinal section of the blade is shown in Figure 7.

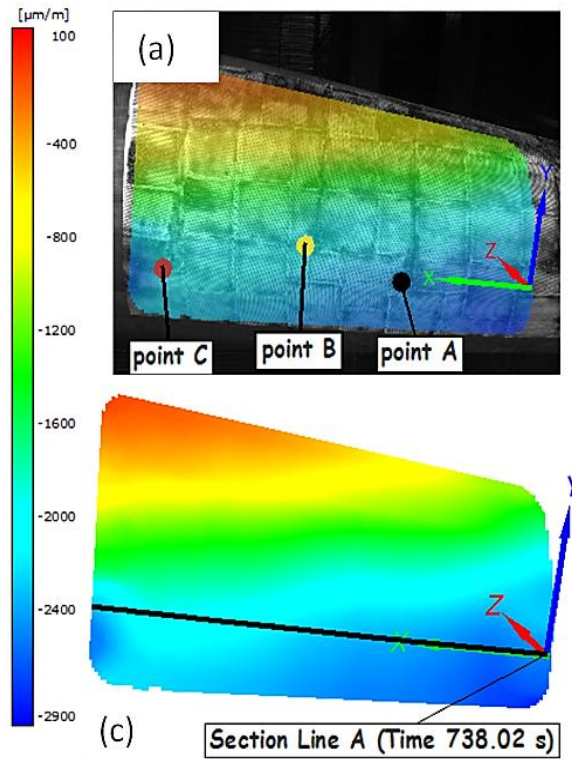

Stage 37

Time $738.02 \mathrm{~s}$
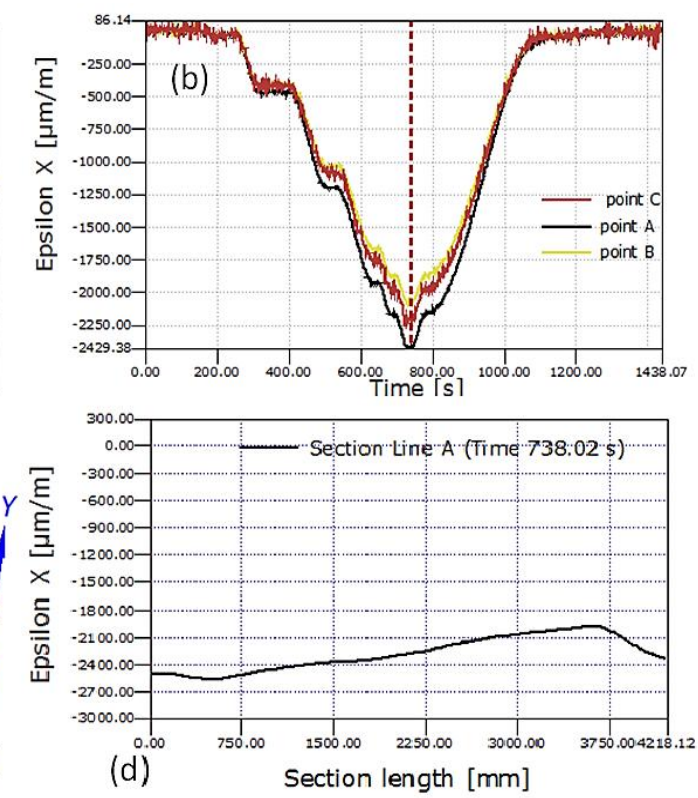

Figure 7. Measurement displaying strain distribution of the blade along the low pressure surface (a) overlay of the blade image and spatial strain contour; (b) a comparison of in-plane strain values at various locations; (c) spatial in-plane strain contour near the blade root in the x-direction; (d) strain section line along the length of the blade (root shown on left).

The strain measurement has some noise that may be attributed to poor speckle patterning, uneven light distribution over the measurement surface, and calibration errors. The accuracy of strain estimation may be improved by smoothing the computed displacement field first and subsequently differentiating to calculate strains. Bing. P and et al. [31] used two dimensional Savitzky-Golay digital differentiator to compute strain from measured full-field displacement fields. In this study, the displacement components were averaged over a finite area (computation area) prior to estimating the strain. Then, the strain components were derived from discrete central difference derivatives of displacement. The size of computation area includes a collection of facets (4 facets) to compute the strain values. Figure $3 \mathrm{~b}$ shows the facet size and facet steps used to process the data and extract strain values.

\subsection{Fatigue Test}

Currently, over the course of multi-month fatigue test, very little information about the spatial distribution of blade deflection and twisting is known. In order to understand the dynamics of a blade as it ages and fatigue damage is induced, it is important to monitor the distributed strain, deflections, and deformations. Fatigue test within the test facility is performed using a hydraulic shaker that can excite the blade at multiple resonant frequencies. By performing optical measurement over a large area of a utility scale wind turbine blade, it would be possible to develop a new approach for structural health monitoring of wind turbine blades. This sensor information will be useful to understand and create prediction tools in determining when blades are either damaged or about to fail. Fatigue testing is highly accelerated life testing to simulate what a wind turbine blade experiences in its life time on a wind turbine [32]. In order to meet the fatigue testing requirements of the International Electro-Technical Commission standard (IEC 61400-23), a Ground-based Resonant Excitation (GREX) system was used to apply automated cyclic loading to the utility-scale blade 
at its first resonant frequency. The hydraulic shaker of the GREX applies loads at a single station on the blade in the flap-wise direction. While exciting the blade in flap wise direction, the in-plane strain and out-of-plane displacement were computed. The load was transmitted by means of the hydraulic shaker in the mid-section of the blade as shown in Figure 8.

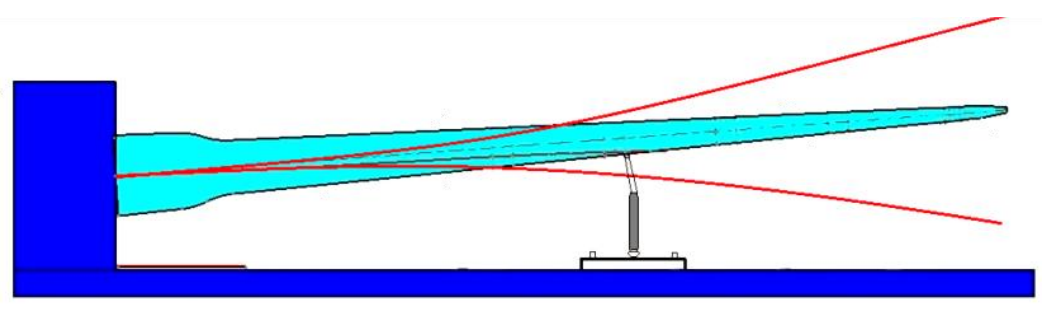

Figure 8 . Fatigue testing of utility scale wind turbine blade.

Using ARAMIS, the DIC measurement was carried out on the AMI blade subjected to fatigue testing. An optical measurement was performed on the same area of the blade during the fatigue test. Although 445 images were taken during the test, only 215 were used which represents 30 seconds of data and approximately 30 blade oscillations. The objective of the experiment is to demonstrate the accuracy of the DIC measurement for the dynamic tests. Only a few seconds of data are necessary to evaluate the capability of the full-field measurement during the fatigue test, and for convenience data is only analyzed over 30 seconds (see figure 9). The collected data was processed and strain distributions along with displacement on the low pressure side of the blade were quantified. The overall results depicted in Figure 9 and 10 indicate that the proposed measurement approach can clearly identify the out of plane displacement as well as strain distribution over the area of interest.

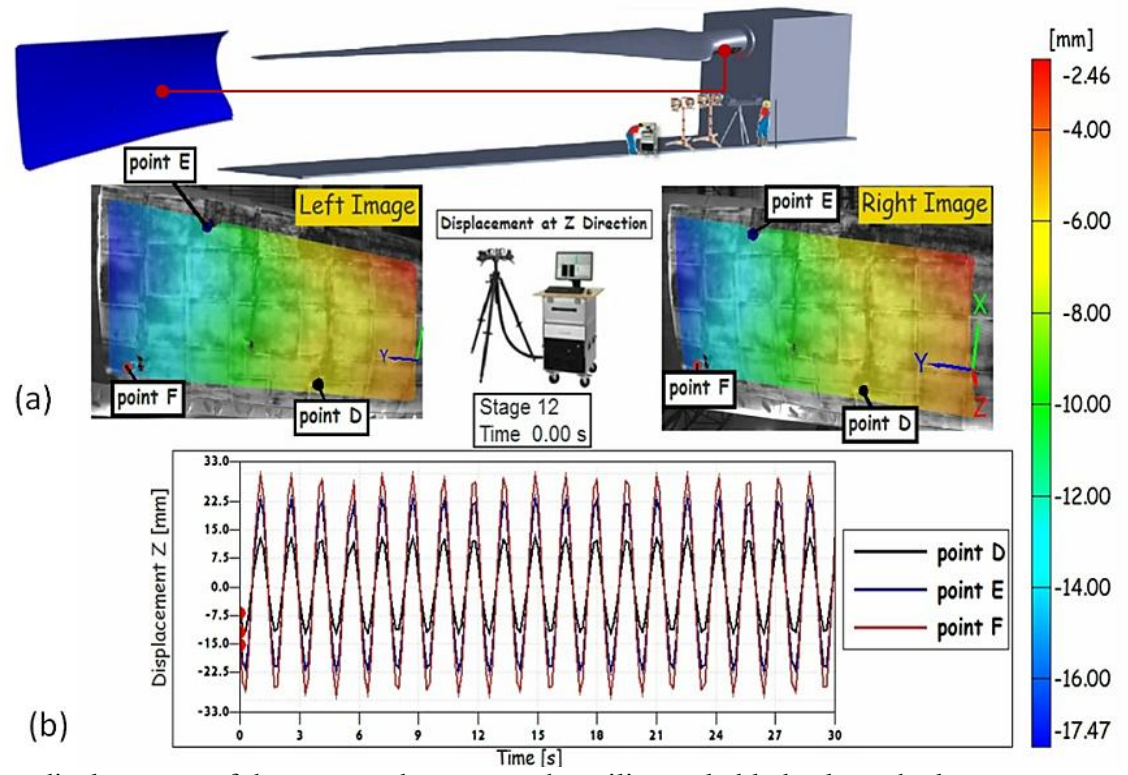

Figure 9. Out of plane displacement of the patterned area over the utility scale blade along the low pressure surface: (a) the spatial displacement contour on the speckled surface; (b) out of plane displacement values at three different points on the measurement surface. 


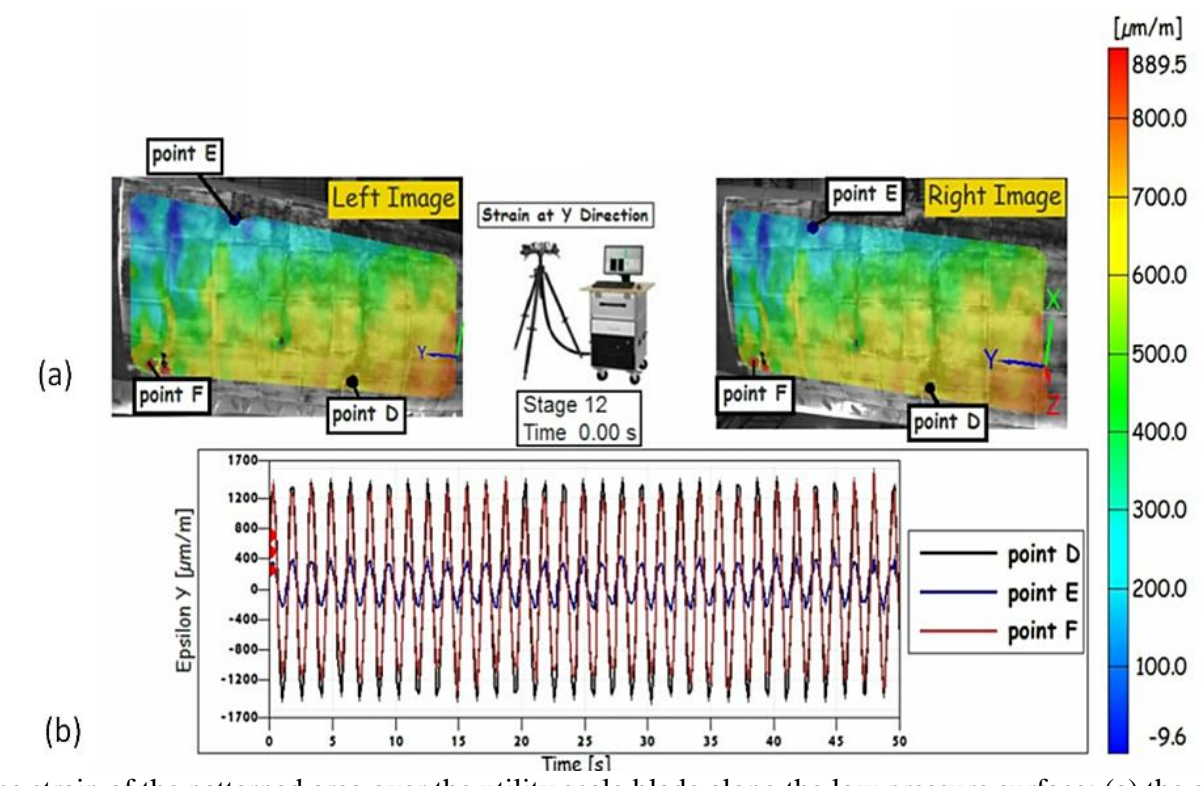

Figure 10. In-plane strain of the patterned area over the utility scale blade along the low pressure surface: (a) the spatial elastic strain contour on the speckled surface; (b) strain values at three different points on the measurement surface.

\section{DYNAMIC SURFACE STITCHING OF LARGE SCALE STRUCTURES}

Because of a sensor's field of view limitation, large scale and complex objects need to be measured from many different viewing directions. Therefore, a multi-camera system in conjunction with spatial point cloud stitching are proposed here to measure 3D displacement of large-scale wind turbine blades. The theoretical background of the stitching technique and operating modal analysis method used in the current work is discussed herein.

\subsection{Calibration Procedure for Multi-camera 3D Point Tracking (3DPT)}

There are two common approaches that can be adopted for the calibration of a multi-camera system. The first calibration approach is to consider a multi stereo-vision system as a single measurement system and calibrate all the conventional stereo systems at the same time [30]. To perform such a calibration, all cameras should have a common field of view with the master camera. In practice this calibration approach is difficult to satisfy. However, the calibration method used in this experiment is based on calibrating each camera individually by knowing the focal length and sensor size to obtain intrinsic parameters (e.g. lens distortion, relative pixel size for the field of view). Stereo calibration is carried out for each conventional stereo 3DPT system to obtain extrinsic (set of rotations and translations required to relate the master and slave cameras) parameters (as shown in Figure 11) to determine the relative position of the cameras. Afterwards, each individual measurement including the geometries and displacement fields are then converted to the common coordinate system. The transformation matrices that relate the world coordinates system to the origin (pinhole) of the camera 1 and camera 2 systems can be presented as follows [33]:

$$
\begin{aligned}
& \left\{\begin{array}{l}
\mathrm{X}_{\mathrm{c} 1} \\
\mathrm{Y}_{\mathrm{c} 1} \\
\mathrm{Z}_{\mathrm{c} 1}
\end{array}\right\}=\left[\mathrm{R}_{\mathrm{A} 01}\right]\left\{\begin{array}{c}
\mathrm{X}_{\mathrm{g}} \\
\mathrm{Y}_{\mathrm{g}} \\
\mathrm{Z}_{\mathrm{g}}
\end{array}\right\}+\left\{\begin{array}{c}
\mathrm{T}_{\mathrm{X}_{\mathrm{A} 01}} \\
\mathrm{~T}_{\mathrm{Y}_{\mathrm{A} 01}} \\
\mathrm{~T}_{\mathrm{Z}_{\mathrm{A} 01}}
\end{array}\right\} \Rightarrow\left\{\begin{array}{c}
\mathrm{X}_{\mathrm{c} 1} \\
\mathrm{Y}_{\mathrm{c} 1} \\
\mathrm{Z}_{\mathrm{c} 1} \\
1
\end{array}\right\}=\left[\begin{array}{cc}
\left\{\left[\mathrm{R}_{\mathrm{A} 01}\right]\right\}_{3 \times 3} & \left\{\left\{\mathrm{~T}_{\mathrm{A} 01}\right\}\right\}_{3 \times 1} \\
\left\{\{0\}^{\mathrm{T}}\right\}_{1 \times 3} & \{1\}_{1 \times 1}
\end{array}\right] \times\left\{\begin{array}{c}
\mathrm{X}_{\mathrm{g}} \\
\mathrm{Y}_{\mathrm{g}} \\
\mathrm{Z}_{\mathrm{g}} \\
1
\end{array}\right\} \\
& \left\{\begin{array}{l}
\mathrm{X}_{\mathrm{c} 2} \\
\mathrm{Y}_{\mathrm{c} 2}
\end{array}\right\}=\left[\mathrm{R}_{\mathrm{A} 02}\right]\left\{\begin{array}{c}
\mathrm{X}_{\mathrm{g}} \\
\mathrm{Y}_{\mathrm{g}} \\
\mathrm{Z}_{\mathrm{g}}
\end{array}\right\}+\left\{\begin{array}{c}
\mathrm{T}_{\mathrm{X}_{\mathrm{A} 02}} \\
\mathrm{~T}_{\mathrm{Y}_{\mathrm{A} 02}} \\
\mathrm{~T}_{\mathrm{Z}_{\mathrm{A} 02}}
\end{array}\right\} \Rightarrow\left\{\begin{array}{c}
\mathrm{X}_{\mathrm{c} 2} \\
\mathrm{Y}_{\mathrm{c} 2} \\
\mathrm{Z}_{\mathrm{c} 2} \\
1
\end{array}\right\}=\left[\begin{array}{cc}
\left\{\left[\mathrm{R}_{\mathrm{A} 02}\right]\right\}_{3 \times 3} & \left\{\left\{\mathrm{~T}_{\mathrm{A} 02}\right\}\right\}_{3 \times 1} \\
\left\{\{0\}^{\mathrm{T}}\right\}_{1 \times 3} & \{1\}_{1 \times 1}
\end{array}\right] \times\left\{\begin{array}{c}
\mathrm{X}_{\mathrm{g}} \\
\mathrm{Y}_{\mathrm{g}} \\
\mathrm{Z}_{\mathrm{g}} \\
1
\end{array}\right\}
\end{aligned}
$$

$R_{A 01}$ and $R_{A 02}$ are rotation matrices that rotate the world coordinate system to align with the left camera (camera 1 ) and the right camera (camera 2) in the stereo-vision A. Vectors $\mathrm{T}_{\mathrm{A} 01}$ and $\mathrm{T}_{\mathrm{A} 02}$, translate the world coordinate system to the 
origin of the camera 1 and camera 2 in stereo-vision A, respectively. The origin of coordinate system for stereo-vision system $\mathrm{A}$ is defined relative to the master camera (camera 1). The relationship between the coordinate system of the master and slave cameras in stereo-vision system $\mathrm{A}$ is given as:

$$
\left\{\begin{array}{c}
X_{c 2} \\
Y_{c 2} \\
Z_{c 2} \\
1
\end{array}\right\}=\left[\begin{array}{cc}
\left\{\left[R_{A 12}\right]\right\}_{3 \times 3} & \left\{\left\{T_{A 12}\right\}\right\}_{3 \times 1} \\
\left\{\{0\}^{T}\right\}_{1 \times 3} & \{1\}_{1 \times 1}
\end{array}\right] \times\left\{\begin{array}{c}
X_{c 1} \\
Y_{c 1} \\
Z_{c 1} \\
1
\end{array}\right\}
$$

where $\left[\mathrm{R}_{\mathrm{A} 12}\right]$ and $\left\{\mathrm{T}_{\mathrm{A} 12}\right\}$ are the rotation and translation matrices that can be used to define the position of an object relative to the master camera (camera 1 in stereo-vision system A). Independent calibration of each camera is performed using the standard procedure to determine radial lens distortion and intrinsic perspective parameters for all the cameras. The intrinsic parameters are used to form rotation and translation matrices $\left(\left[\mathrm{R}_{\mathrm{A} 01}\right],\left[\mathrm{R}_{\mathrm{A02}}\right],\left\{\mathrm{T}_{\mathrm{A} 01}\right\}\right.$, and $\left.\left\{\mathrm{T}_{\mathrm{A} 02}\right\}\right)$. The knowledge of each camera's position relative to each other in stereo-vision A can be obtained through the extrinsic parameters (i.e. the relative position and orientation of each camera w.r.t. each other). To obtain the calibration, a calibration crossbar is positioned in front of the both cameras and triangulation is used to obtain the extrinsic parameters.

Calibrating camera 3 and camera 4 in stereo-vision B yield the knowledge of imaging parameters for each optical sensor (intrinsic parameters) and the relative orientation and position of the optical sensors (camera 3 and 4) with respect to each other (extrinsic parameters) in stereo-vision B. Equation 4 represents the relation between master and slave cameras in stereo-vision B.

$$
\left\{\begin{array}{c}
X_{c 4} \\
Y_{c 4} \\
Z_{c 4} \\
1
\end{array}\right\}=\left[\begin{array}{cc}
\left\{\left[R_{B 12}\right]\right\}_{3 \times 3} & \left\{\left\{T_{B 12}\right\}\right\}_{3 \times 1} \\
\left\{\{0\}^{T}\right\}_{1 \times 3} & \{1\}_{1 \times 1}
\end{array}\right] \times\left\{\begin{array}{c}
X_{c 3} \\
Y_{c 3} \\
Z_{c 3} \\
1
\end{array}\right\}
$$

Figure 11 shows the extrinsic parameters that can be estimated by calibration of each individual stereo-vision system. The components of the rotation matrices $\left(\left[\mathrm{R}_{\mathrm{A} 12}\right],\left[\mathrm{R}_{\mathrm{B} 12}\right]\right)$ and translation matrices $\left(\left\{\mathrm{T}_{\mathrm{A} 12}\right\},\left\{\mathrm{T}_{\mathrm{B} 12}\right\}\right)$ in Equation 3 and 4 depend on the extrinsic parameters of the conventional stereo-vision systems [24]. Using the common points that are visible by different stereo-vision systems (stereo-vision A and B), measured point clouds can be related to each other.

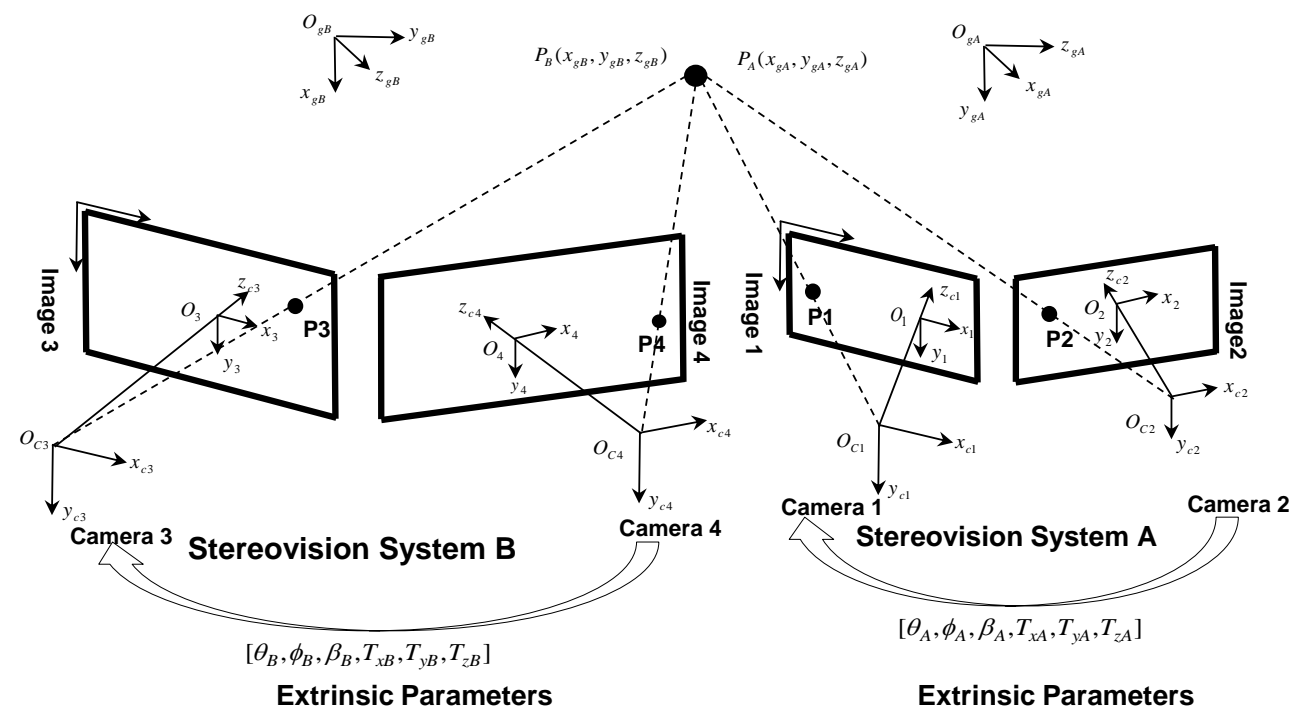

Figure 11. A schematic describing the transformation between cameras in a multiple stereo-vision system. Global coordinate system is assumed to be the same for both cameras. 


\section{VALIDATION OF THE PROPOSED MULTI-CAMERA 3DPT METHOD}

A measurement to experimentally validate the proposed approach of a multi-camera 3DPT was performed on a 2.3meter cantilevered wind turbine blade, Skystream $4.7^{\mathrm{TM}}$ shown in Figure 12. Two stereo-vision systems composed of four synchronized complementary metal-oxide semiconductor (CMOS) cameras were used in the experimental setup. As shown in Figure 12, a pair of four megapixel $(2048 \times 2048$ pixel CMOS sensor) PHOTRON high-speed cameras (stereo-vision B) and a pair of twelve-megapixel cameras (4096 × 3072 pixel CMOS sensor, stereo-vision A) equipped with 24-mm lenses were used for the measurement. Stereo-vision system A was placed approximately 1.3-meters away from the turbine blade and stereo-vision system B was placed approximately 1.6 meters away from the blade. Figure 13 shows the fields of view of the cameras and the overlapped area covered by both stereo-vision systems. The left half of the blade was covered by stereo-vision B while the right half was covered by stereo-vision A. All four cameras were synchronized to capture the motion of the cantilevered blade at the same time. A collection of optical targets (132) was mounted to the blade and the fixture to measure the in-plane and out of plane displacements of the blade. A one-meter calibration crossbar was used to calibrate each individual stereo-vision system. Calibration is performed on the effective measurement volume to obtain the extrinsic, intrinsic parameters and radial distortion coefficients for each vision system. The perspective intrinsic parameters describe the properties of the camera including focal length, image sensor format, and principle format. The extrinsic parameters determine the relative position of the cameras with respect to one another. For DIC and 3DPT (using the Aramis ${ }^{\mathrm{TM}}$ and Pontos $^{\mathrm{TM}}$ systems) the calibration is performed identically [30, 34].

The accuracy of the multi-camera 3DPT measurement, apart from the standard stereo 3DPT itself, also is influenced by the stitching of two different displacement fields. The overlap area or the common field of view between two stereovision systems, shown in Figure 12, provides the link between two displacement fields and makes them feasible to be stitched by transforming the reference points. However, as the reference points (see Figure 13a) include some uncertainties associated with the systematic error of standard stereo-photogrammetry, these uncertainties propagate during the transformation operation. The uncertainties of the 3DPT results stem from different sources such as statistical noise of the gray level values, different illumination conditions for different cameras, image contrast, and size of the speckle pattern. [35, 36]. Within the current paper, three points are used to find the rotation matrix that can align two point clouds. Any uncertainties originated from 3DPT measurement of those three points will propagate through the transformation operation. 


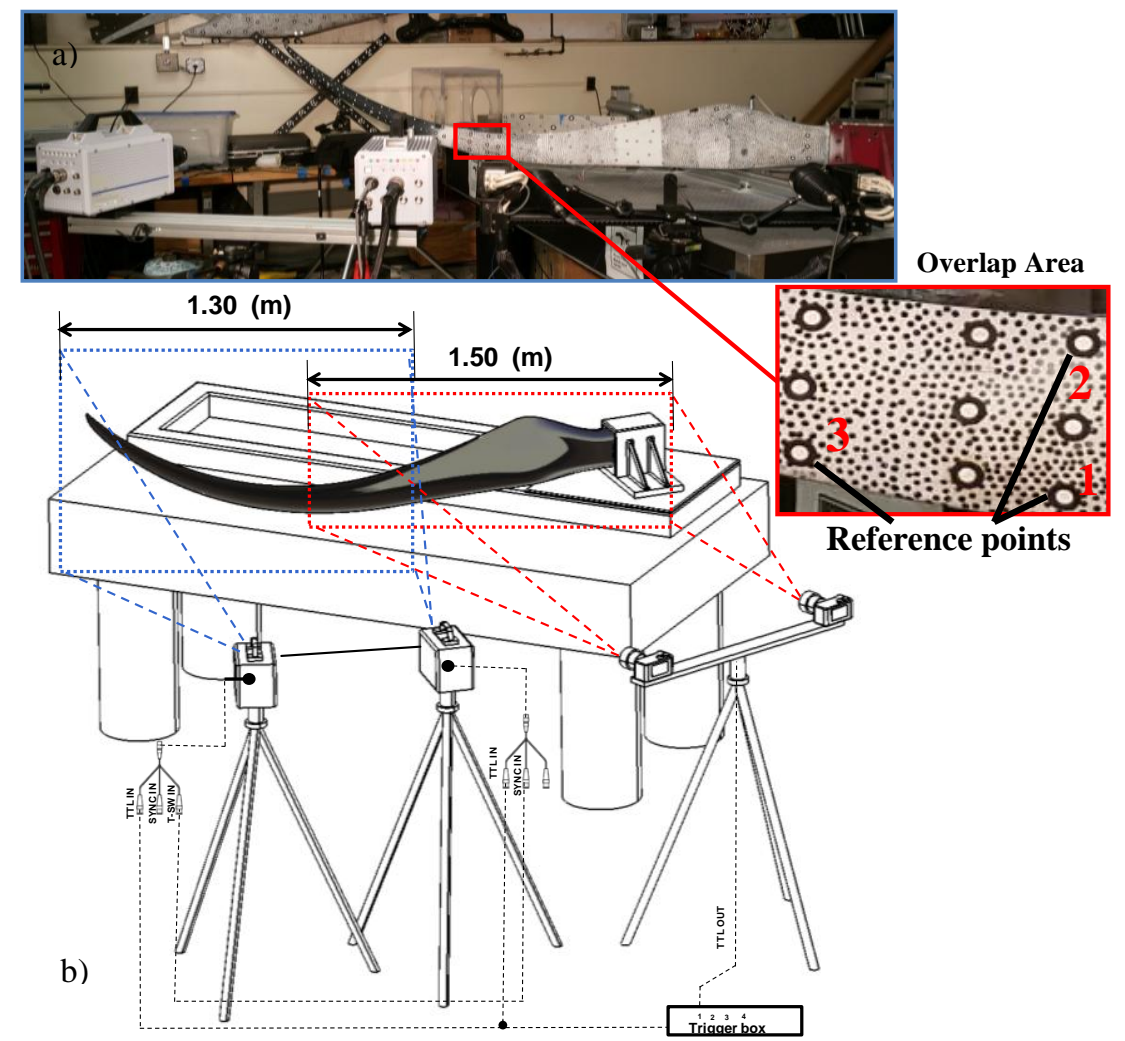

Figure 12. Multi-camera setup with the two synchronized stereo-vision systems and the cable routing diagram; a) measurement systems including four CMOS cameras and the overlapped area shown with red solid box, b) schematic illustration of the test article and the fields of views from the two stereo-vision systems."

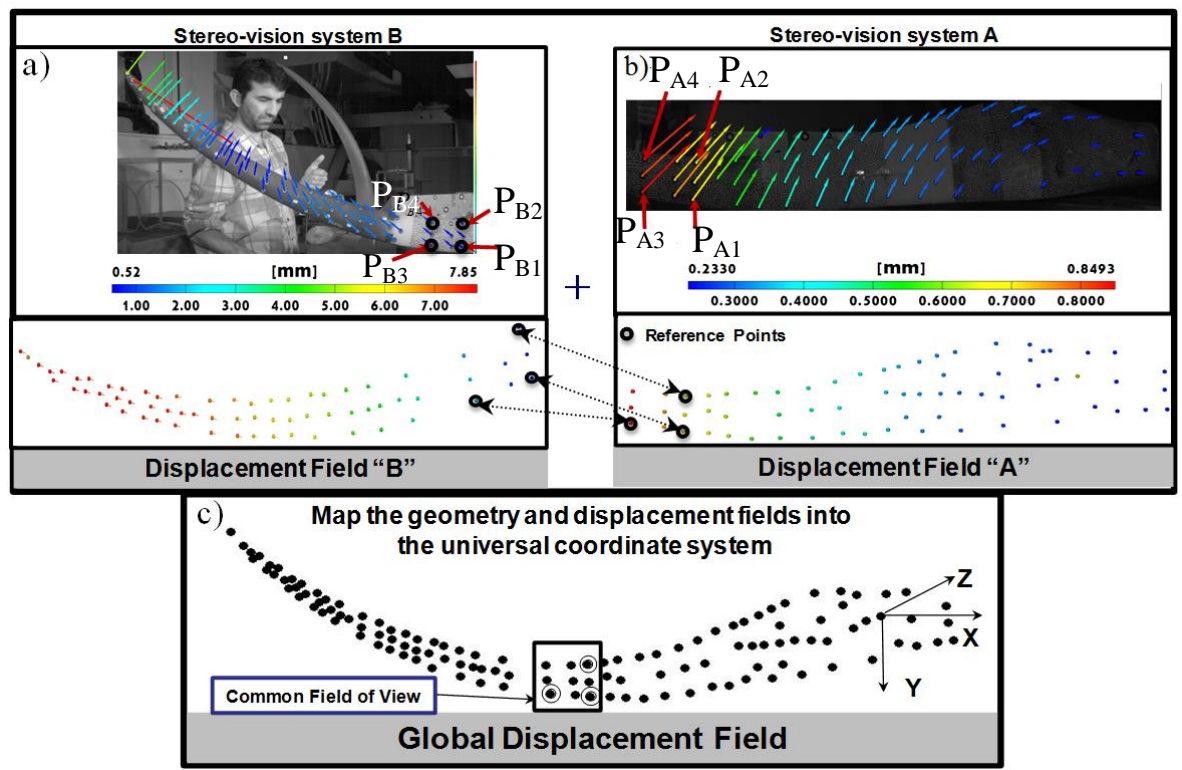

Figure 13. Views from two stereo-vision systems ; a) the displacement field measured by stereo-vision A, b) the displacement field measured by stereo-vision B, c) a view showing the optical targets on the entire blade formed by stitching from the two views. 
Because there are at least three corresponding points (PointP_A1/P_B1, P_A2/P_B2, and P_A3/P_B3 shown in Figure 11) in the overlapping area, the method used to find the appropriate transformation matrix minimizes the sum of the Euclidean distances between these common points. This corresponds to a least-square minimization problem that can be solved using singular value decomposition (SVD). The procedure of estimating the transformation matrix using SVD can be broken down into the following sequential steps: (1) the centroids of the common points in two point clouds are calculated, (2) both sets of common points using the centroids are re-centered, (3) the cross-correlation matrix between the two centered point clouds are computed, and (4) the singular value decomposition is used to find optimal rotation matrix.

In the displacement field $\mathrm{A}$, Point $\mathrm{P}_{\mathrm{A} 1}, \mathrm{P}_{\mathrm{A} 2}$, and $\mathrm{P}_{\mathrm{A} 3}$ are the reference points that have correspondence in the displacement field $\mathrm{B}$ (Point $\mathrm{P}_{\mathrm{B} 1}, \mathrm{P}_{\mathrm{B} 2}$, and $\mathrm{P}_{\mathrm{B} 3}$ ). The purpose of the transformation is to assign a reference point in displacement field $\mathrm{B}$ to one of the reference points in the displacement field $\mathrm{A}$, so that these reference points represent the same physical points in 3D space. For instance, point $\mathrm{P}_{\mathrm{A} 1}$ in the displacement field $\mathrm{A}$ is the correspondent of point $\mathrm{P}_{\mathrm{B} 1}$ in the displacement field B, as shown in Figure 13. To assess the accuracy of the proposed stitching method, the out of plane position of these two corresponding points $\left(\mathrm{P}_{\mathrm{A} 1}\right.$ and $\left.\mathrm{P}_{\mathrm{B} 1}\right)$ in the global coordinate system is compared. The difference of the out of plane displacement of point $\mathrm{P}_{\mathrm{B} 1}$ and $\mathrm{P}_{\mathrm{A} 1}$ was determined to be below the noise floor range of both stereovision systems (see Figure 14.a). Unlike point $\mathrm{P}_{\mathrm{B} 1}$ and $\mathrm{P}_{\mathrm{A} 1}$, point $\mathrm{P}_{\mathrm{B} 4}$ and $\mathrm{P}_{\mathrm{A} 4}$ are not used to stitch the point clouds measured using stereo-vision A and B.

Similar to the matching of the previous points $\left(\mathrm{P}_{\mathrm{B} 1}\right.$ and $\left.\mathrm{P}_{\mathrm{A} 1}\right)$, the positions of two other corresponding points $\left(\mathrm{P}_{\mathrm{B} 4}\right.$ and $\mathrm{P}_{\mathrm{A} 4}$ ) are compared in Figure 15. The average displacement error and the strong correlation between the traces of the common points in the overlapping field of view show that the proposed stitching method can produce accurate results within the range of calibration error of two stereo-vision systems. The time response assurance criterion (TRAC) is used to quantify the correlation between two time traces. TRAC values close to 1.0 indicate very good similarities, where values close to 0.0 indicate minimal or no similarity between the time traces [37]. For the results shown in Figures 14 and 15, the TRAC values indicates the two time traces for the different targets in field of view A and B are almost identical. The average displacement errors and standard deviations of four corresponding points in the overlap area are shown in Table 1. $\Delta \mathrm{x}$ is the displacement error along the blade, $\Delta \mathrm{y}$, is the in-plane (edge-wise) displacement error in edge direction and $\Delta \mathrm{z}$, is the out of plane displacement (flap-wise) error. The results show that the out of plane displacement errors are greater than $200 \mu \mathrm{m}$ with standard deviations less than $22 \mu \mathrm{m}$. The displacement uncertainty of the designed multi-camera system is mostly due to the fact that two stereo-vision systems provide slightly different measurements due to the different calibrations and optical sensors. It was found that the maximum displacement error at the tip is $0.07 \mathrm{~mm}$. 


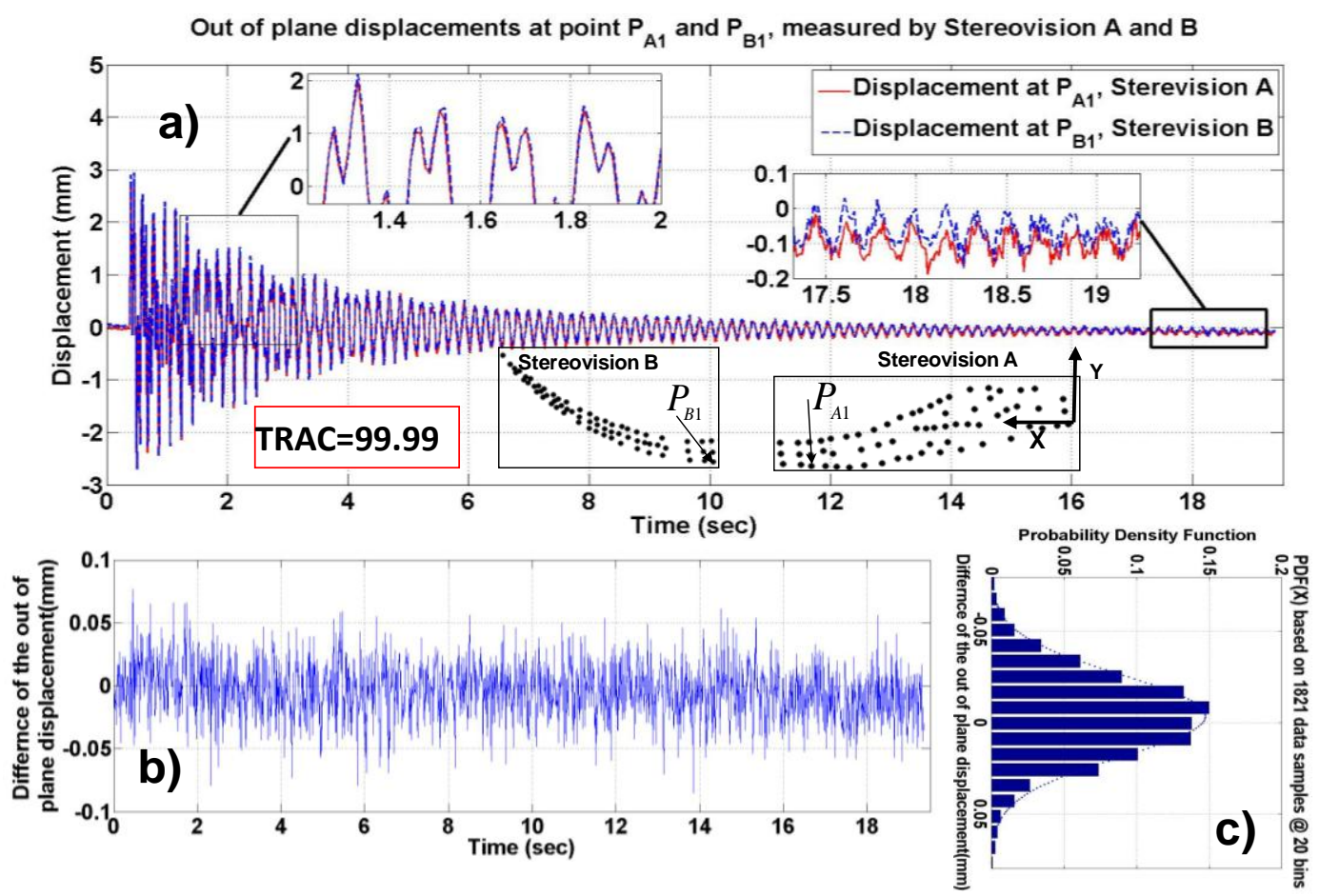

Figure 14. Comparison of the out of plane displacements at points $P_{B 1}$ and $P_{A 1}$; a) displacement at points $P_{B 1}$ and $P_{A 1}$ when the blade is impacted with an impact hammer, $b$ ) the uncertainty of the multi-camera system at the location of two corresponding points $\mathrm{P}_{\mathrm{B} 1}$ and $\mathrm{P}_{\mathrm{A} 1}$, c) the probability density function of displacement error based on the 1821 image stages.

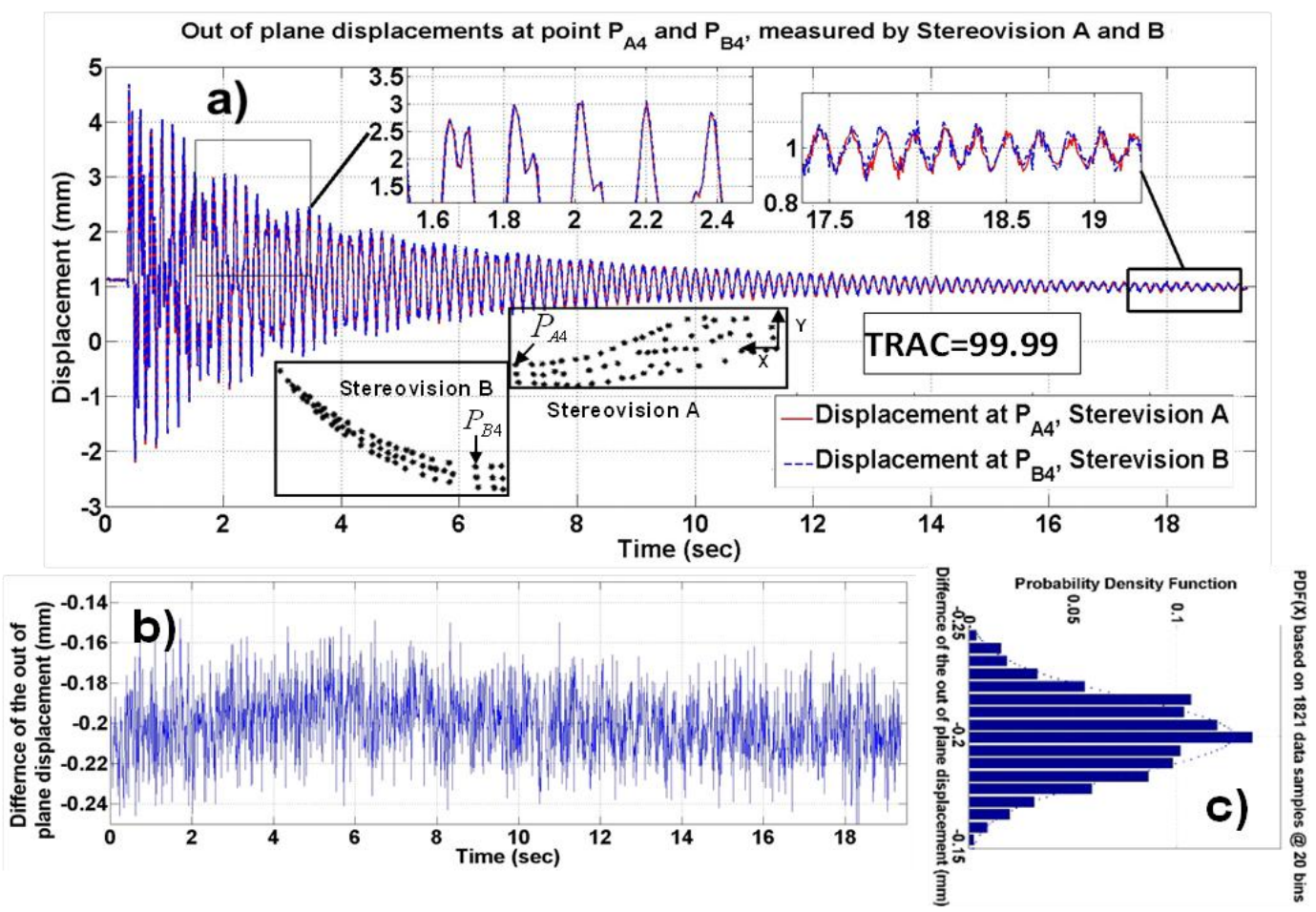

Figure 15. Comparison of the out of plane displacement at points $P_{B 4}$ and $P_{A 4}$; a) displacement at points $P_{B 4}$ and $P_{A 4}$ when the blade is impacted with an impact hammer, b) the uncertainty of the multi-camera system at the location of two corresponding points $P_{B 4}$ and $P_{A 4}$, c) the probability density function of displacement error based on the 1821image stages. 
Table 1. Influence of the reference frame transformation on the displacement measurements in the multi-camera 3DPT system. The difference between the out-of-plane and in-plane displacements of the common points in the overlapped area are quantified.

\begin{tabular}{ccccc}
\hline & Transformation & $\Delta x(\mu m)$ & $\Delta y(\mu m)$ & $\Delta z(\mu m)$ \\
\hline \multirow{4}{*}{$\begin{array}{c}\text { Average } \\
\text { error }\end{array}$} & $\left(\overrightarrow{\boldsymbol{P}_{B 1}}-\overrightarrow{\boldsymbol{P}_{A 1}}\right)$ & 11 & 4 & 3 \\
& $\left(\overrightarrow{\boldsymbol{P}_{B 3}}-\overrightarrow{\boldsymbol{P}_{A 2}}\right)$ & 21 & 12 & 14 \\
& $\left(\overrightarrow{\boldsymbol{P}_{B 4}}-\overrightarrow{\boldsymbol{P}_{A 4}}\right)$ & 65 & 25 & 177 \\
\hline \multirow{4}{*}{ Standard } & $\left(\overrightarrow{\boldsymbol{P}_{B 1}}-\overrightarrow{\boldsymbol{P}_{A 1}}\right)$ & $\mathbf{7 1}$ & 12 & 200 \\
deviation & $\left(\overrightarrow{\boldsymbol{P}_{B 2}}-\overrightarrow{\boldsymbol{P}_{A 2}}\right)$ & 11 & 47 & 22 \\
& $\left(\overrightarrow{\boldsymbol{P}_{B 3}}-\overrightarrow{\boldsymbol{P}_{A 3}}\right)$ & 18 & 51 & $\mathbf{3 4}$ \\
& $\left(\overrightarrow{\boldsymbol{P}_{B 4}}-\overrightarrow{\boldsymbol{P}_{A 4}}\right)$ & 12 & 38 & 17 \\
\hline
\end{tabular}

\section{EXTRACTING OPTICALLY MEASURED SHAPES USING OPERATIONAL MODAL ANALYSIS (OMA)}

Operational modal analysis (OMA) is a structural dynamic testing procedure that estimates modal parameters from only the structural response (i.e. unknown input). Although the use of OMA dates back to the 1930s, the most significant advances in OMA testing techniques occurred since the early 1990's. OMA has several advantages over traditional experimental modal analysis and has become attractive because: (1) no elaborate excitation equipment is needed to perform the test; (2) the approach is not as labor intensive (i.e. low cost) as it relies on the naturally induced vibration that occur during operation; and (3) the dynamic characteristic of the structure is extracted in its true boundary conditions [38]. Experimental mode shapes depend on the inherent properties of the structure; therefore, experimentally estimated mode shapes from the input-output measurement characterize the resonant vibration of the structure. However, operating deflection shapes depend on the force level and will be subjected to change if the input forces changes. Therefore, operating deflection shape can be associated with either forced or resonant vibration. Within the current work, the impulsive input is employed to excite all of the mode shapes in the frequency band of the interest. Because the power spectral density of the impulse excitation is almost flat for the lower frequencies and similar to the power spectral density of white noise, the distribution of the input energy among the frequencies of interest is approximately uniform. It is worth mentioning that, under the assumption of white noise input, output-only system identification methods use the output spectra to identify modal parameters.

A multi-camera 3DPT system was used to measure 3D dynamic displacement response of optical targets attached to the blade. Using LMS operational PolyMAX ${ }^{\mathrm{TM}}$ [39], four stable poles and their corresponding mode shapes were identified as shown in Figure 16. The PolyMAX method has been well accepted due its superior performance in estimating modal parameters[40, 41]. This method is a further evolution of the least-squares complex frequency-domain (LSCF) estimation method. The most important advantage of the LSCF estimator over the available and widely applied parameter estimation techniques is clear stabilization diagrams. The Operational PolyMAX, derived from PolyMAX [40], is a frequency-domain output-only system identification that requires output spectra as primary data to extract modal parameters from response-only measurements. The blade was excited by using impulse input with a hammer. The first, second, and fourth mode shapes are flap-wise bending modes. The third mode, however, is an edgewise mode. The input force applied by the hammer was not measured; therefore, the results presented are operating mode shapes, not mode shapes. Figure 16 shows the optically measured operating modes below $50 \mathrm{~Hz}$ extracted using operational modal analysis. Because input energy of the impulse force is widespread across the frequency domain, most of the modes are excited exactly as in an EMA test. For large structures, a typical modal hammer can only excite the structure with a relatively small amount of energy, so it may not generate enough mechanical response to be measured by the optical system. Therefore, conducting optically-based modal analysis on large-scale structures such as a utility-scale blade requires designing a very large impact hammer that may be difficult to implement. Because, output-only system identification does not require any controlled excitation, impact testing without controlled force can streamline the structural evaluation of large structures such as utility-scale blades. For the modal test performed in the lab on the scale 
wind turbine blade a hammer was used for convenience to excite all the modes of interest. Future experiments will quantify the difference in using OMA and EMA to process the data on the turbine blade to extract damping and mode shapes at the laboratory scale. A validated solid finite element model of the Skystream $4.7^{\mathrm{TM}}$ was used to evaluate the accuracy of the operating mode shapes extracted using the multi-camera 3DPT system [3, 42]. A more detailed description about the validation of the finite element (FE) model can be found in [43]. A comparison between the FE and experimental mode shapes was performed using the modal assurance criterion (MAC). Strong similarities are seen between the FE and test model for most of the modes as shown in Table 2. High MAC values for both flap-wise and edgewise operating mode shapes reveals the potential of the proposed approach to measure operating shapes of the large utility scale wind turbine blades. However, because the fixture holding the blade could not provide a fully built-in boundary condition, there is an $8 \%$ difference between the test results and FE model for the first mode of vibration. The difference in the natural frequencies for the higher order modes is much smaller. It should be noted that the FE model has already been validated using conventional modal analysis approaches in several published works [42, 44].
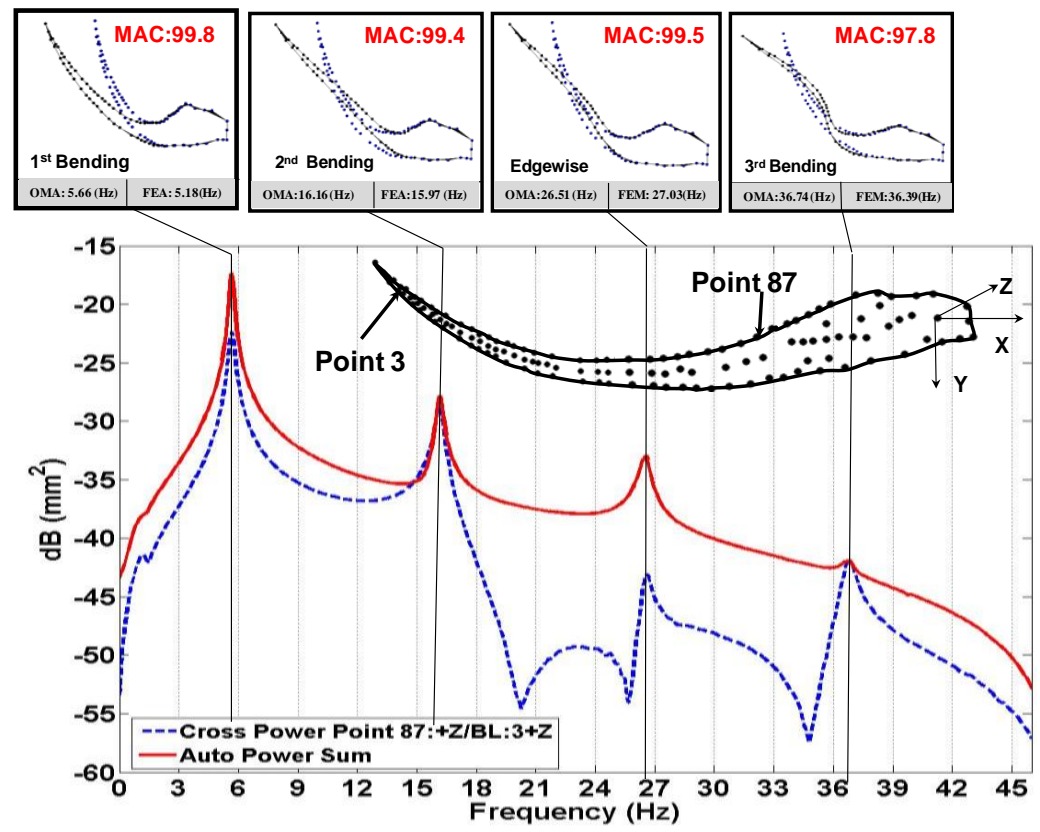

Figure 16. Stability diagram showing the sum of cross power spectra dominated by four flexible modes and the extracted shape information of the cantilevered blade (curve fit using LMS Operational PolyMAX ${ }^{\mathrm{TM}}$ ). 
Table 2. Modal assurance criterion (MAC) comparison and frequency percent difference between experimental frequencies and FE model mode shapes. Red circles indicate the position of the targets in each operating mode shapes of the blade.

\begin{tabular}{|c|c|c|c|c|c|}
\hline & $\begin{array}{l}\text { FEM. } \\
(\mathrm{Hz})\end{array}$ & $\begin{array}{l}\text { OMA. } \\
\text { (HZ) }\end{array}$ & $\begin{array}{l}\text { Diff. } \\
(\%)\end{array}$ & $\begin{array}{c}\text { MAC } \\
(\%) \\
\end{array}$ & Mode Shape Pairs \\
\hline 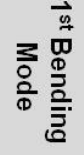 & 5.18 & 5.66 & 8.51 & 99.8 & \\
\hline 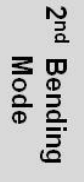 & 15.97 & 16.16 & 1.21 & 99.4 & \\
\hline 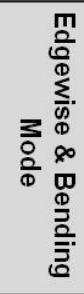 & 27.03 & 26.51 & 1.96 & 99.5 & \\
\hline 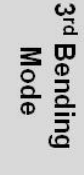 & 36.39 & 36.74 & 0.95 & 97.8 & \\
\hline
\end{tabular}

\section{CONCLUSIONS}

This paper tries to identify the drawbacks associated with a conventional 3D DIC system for large areas and extends the capabilities of 3DPT system by introducing a multi-camera 3DPT system in which data obtained with conventional 3DPT systems are stitched together in a universal coordinate system. This has the potential to be performed with lower cost per test and obtained in less time without the necessary extensive strain gage wiring and with far great spatial resolution for strain measurements, thereby helping to streamline the certification/testing process. It was shown that the technique can be used to extend the field of view of a standard 3DPT system without losing significant resolution and accuracy. Additionally, the merit of the proposed multi-camera system was demonstrated by using two stereo systems in conjunction with output-only system identification to identify operating mode shapes as well as natural frequencies of a wind turbine blade. This eliminated the need for the accelerometer measurement system with its separate accelerometer attachment and associated cabling system. Furthermore, the accuracy of the data stitching procedure was evaluated to demonstrate the ability of the system for wide-area displacement measurement. The acquired modal data was shown to be very accurate when compared to an earlier validated finite element model.

The proposed measurement procedure eliminates the need for two separate measurement systems for strain and acceleration measurements by leveraging a single system that obtains all the data together. Further studies are needed to evaluate the accuracy of the proposed method to inspect an entire surface of a utility-scale wind turbine blade with more than two camera pairs. In principle, since the stitching approach has been shown to be effective for two camera pairs, it should be able to be extended to three or more sensor pairs. Performing large area monitoring and multi-camera measurement of the small-scale wind turbine blade were basic steps to assess the potentialities of the proposes method; this will pave the way for the development of an optical measurement procedure, capable of monitoring the entire surface of large sized blades. 


\section{ACKNOWLEDGEMENTS}

The authors gratefully appreciate the financial support for this work provided by the Massachusetts Clean Energy Center (CEC), Task Order 13-2. Any opinions, findings, and conclusions or recommendations expressed in this material are those of the authors and do not necessarily reflect the views of Mass CEC. The authors would like to also thank the U.S. Department of Energy's Wind and Water Power Technologies Office (DOE/EERE/WWPTO) for making the Advanced Manufacturing Initiative (AMI) Demonstrator Blade available for this research.

\section{REFERENCES}

[1] M.S. Allen, M.W. Sracic, S. Chauhan, M.H. Hansen, Output-only modal analysis of linear time-periodic systems with application to wind turbine simulation data, Mechanical Systems and Signal Processing, Volume 25, Issue 4, 2011, pp. 1174-1191, doi: http://dx.doi.org/10.1016/j.ymssp.2010.12.018.

[2] C. Chia Chen, L. Jung-Ryul, B. Hyung-Joon, Structural health monitoring for a wind turbine system: a review of damage detection methods, Measurement Science and Technology, Volume 19, Issue 12, 2008, pp. 122001.

[3] J. Baqersad, P. Poozesh, C. Niezrecki, P. Avitabile, Comparison of Modal Parameters Extracted Using MIMO, SIMO, and Impact Hammer Tests on a Three-Bladed Wind Turbine, A Conference and Exposition on Structural Dynamics, Orlando, FL, 2014, pp. 185-197. doi:10.1007/978-3-319-04774-4_19.

[4] O. Saber, F.M. Chester, J.L. Alvarado, Development of a Material-Testing Machine for Study of Friction: Experimental Analysis of Machine Dynamics and Friction of Rock, Experimental Mechanics, Volume 56, Issue 5, 2016, pp. 813-831, doi: 10.1007/s11340-015-0125-y.

[5] A. Zanarini, On the estimation of frequency response functions, dynamic rotational degrees of freedom and strain maps from different full field optical techniques, ISMA, Leuven, Belgium, 2012, pp. 1177-1192.

[6] A. Zanarini, Comparative studies on Full Field FRFs estimation from competing optical instruments, IFToMM ICoEV2015 International Conference on Engineering Vibration, Ljubljana (Slovenia), 2015, pp. 1559-1568.

[7] C.W. Schwingshackl, A.B. Stanbridge, C. Zang, D.J. Ewins, Full-field Vibration Measurement of Cylindrical Structures Using a Continuous Scanning LDV Technique, IMAC-XXV: Conference \& Exposition on Structural Dynamics, Orlando FL, USA, 2007.

[8] J. Baqersad, P. Poozesh, C. Niezrecki, P. Avitabile, Photogrammetry and optical methods in structural dynamics - A review, Mechanical Systems and Signal Processing, Volume 72-75, Issue 1, doi: http://dx.doi.org/10.1016/j.ymssp.2016.02.011.

[9] H.V.d. Auweraer, H. Steinbichler, S. Vanlanduit, C. Haberstok, R. Freymann, D. Storer, V. Linet, Application of stroboscopic and pulsed-laser electronic speckle pattern interferometry (ESPI) to modal analysis problems, Measurement Science and Technology, Volume 13, Issue 4, 2002, pp. 451.

[10] J. Zanarini, Full field ESPI measurements on a plate: challenging experimental modal analysis, IMAC 25, A Conference on Structural Dynamics, Orlando, FL, 2007.

[11] A. Zanarini, Full field experimental modelling in spectral approaches to fatigue predictions, IFToMM ICoEV2015 International Conference on Engineering Vibration, Ljubljana (Slovenia), 2015.

[12] A. Zanarini, Full Field ESPI Vibration Measurements to Predict Fatigue Behavior, International Mechanical Engineering Congress and Exposition, American Society of Mechanical Engineers 2008, pp. 165-174.

[13] A. Zanarini, Fatigue life assessment by means of full field espi vibration measurements, Proceedings of the ISMA2008 Conference, September, 2008, pp. 15-17.

[14] T. Schmidt, J. Tyson, K. Galanulis, Full-field dynamic dispalcement ans strain measurment using advanced 3D image correlation photogrammetry:part 1, Experimental Techniques, Volume 27, Issue 3, 2003, pp. 47-50, doi: 10.1111/j.1747-1567.2003.tb00115.x.

[15] M. Sutton, W. Wolters, W. Peters, W. Ranson, S. McNeill, Determination of displacements using an improved digital correlation method, Image and Vision Computing, Volume 1, Issue 3, August 1983, pp. 133-139.

[16] M. Sutton, C. Mingqi, W. Peters, Y. Chao, S. McNeill, Application of an optimized digital correlation method to planar deformation analysis, Image and Vision Computing, Volume 4, Issue 3, August 1986, pp. 143-150.

[17] T.C. Chu, W.F. Ranson, M.A. Sutton, Applications of digital-image-correlation techniques to experimental mechanics, Experimental Mechanics, Volume 25, Issue 3, September 1985, pp. 232-244.

[18] M. Ozbek, D.J. Rixen, Optical measurements and operational modal analysis on a large wind turbine: Lessons learned, Jacksonville, FL, March 2011, pp. 257-276. 
[19] M. Ozbek, D.J. Rixen, O. Erne, G. Sanow, Feasibility of monitoring large wind turbines using photogrammetry, Energy, Volume 35, Issue 12, December 2010, pp. 4802-4811, doi: 10.1016/j.energy.2010.09.008.

[20] M. Ozbek, F. Mengt, D.J. Rixen, M.J.L. Van Tooren, Identification of the dynamics of large wind turbines by using photogrammetry, 28th IMAC, A Conference on Structural Dynamics, Jacksonville, FL, February 2010, pp. 351-359.

[21] M. Ozbek, D.J. Rixen, Operational modal analysis of a $2.5 \mathrm{MW}$ wind turbine using optical measurement techniques and strain gauges, Wind Energy, Volume 16, Issue3, February 2012, pp. 367-381, doi: 10.1002/we.1493.

[22] U.S. Paulsen, T. Schmidt, O. Erne, Developments in large wind turbine modal analysis using point tracking videogrammetry, 28th IMAC, A Conference on Structural Dynamics, Jacksonville, FL, June 2011, pp. 187-198.

[23] P. Poozesh, J. Baqersad, C. Niezrecki, E. Harvey, R. Yarala, Full Field Inspection of a Utility Scale Wind Turbine Blade Using Digital Image Correlation, CAMX, Orlando, FL, 2014.

[24] F. Chen, X. Chen, X. Xie, X. Feng, L. Yang, Full-field 3D measurement using multi-camera digital image correlation system, Optics and Lasers in Engineering, Volume 51, Issue 9, September 2013, pp. 1044-1052.

[25] Y. Wang, P. Lava, S. Coppieters, P.V. Houtte, D. Debruyne, Application of a multi-camera stereo DIC set-up to assess strain fields in an Erichsen test: Methodology and validation, Strain, Volume 49, Issue 2, April 2013, pp. 190-198. [26] P. Poozesh, J. Baqersad, C. Niezrecki, P. Avitabile, A Multi-Camera Stereo DIC System for Extracting Operating Mode Shapes of Large Scale Structures, Costa Mesa, CA, June 2015.

[27] T.N. Nguyen, J.M. Huntley, R.L. Burguete, C.R. Coggrave, Multiple-view shape and deformation measurement by combining fringe projection and digital image correlation, Strain, Volume 48, Issue 3, June 2012, pp. 256-266.

[28] B. LeBlanc, C. Niezrecki, P. Avitabile, J. Chen, J. Sherwood, Damage detection and full surface characterization of a wind turbine blade using three-dimensional digital image correlation, Structural Health Monitoring, Volume 12, Issue 5-6, September 2013, pp. 430-439.

[29] M. Malesa, K. Malowany, M. Kujawińska, Multi-camera DIC system with a spatial data stitching procedure for measurements of engineering objects, Photonics Letters of Poland, Volume 6, Issue 4, 2014, pp. 157-159.

[30] ARAMIS v6.3, GOM mbH, Braunschweig, Germany 2011.

[31] H.M.X. Bing Pan, Tao Hua, Wei Ning Wang, Yan Fang, Displacement Smoothing and Strain Estimation Using Savitaky-Golay Filters in Digital Image Correlation, Key Engineering Materials, Volume 326, Issue 328, December 2006, pp. 135-138, doi: 10.4028/www.scientific.net/KEM.326-328.135

[32] M. Helfrick, An Investigation of 3D Digital Image Correlation for Structural Health Monitoring and Vibration Measurement, Mechanical Engineering Department,Master Thesis, University of Massachusetts Lowell, 2008.

[33] M.A. Sutton, J.J. Orteu, H. Schreier, Image correlation for shape, motion and deformation measurements: basic concepts, theory and applications, Springer Science \& Business Media, 2009.

[34] PONTOS v6.3, GOM mbH, Braunschweig, Germany 2011.

[35] E. Zappa, A. Matinmanesh, P. Mazzoleni, Evaluation and improvement of digital image correlation uncertainty in dynamic conditions, Optics and Lasers in Engineering, Volume 59, 2014, pp. 82-92, doi: http://dx.doi.org/10.1016/j.optlaseng.2014.03.007.

[36] G. D'Emilia, L. Razzè, E. Zappa, Uncertainty analysis of high frequency image-based vibration measurements, Measurement, Volume 46, Issue8, 2013, pp. 2630-2637, doi: http://dx.doi.org/10.1016/j.measurement.2013.04.075.

[37] T.V. Zandt, Development of efficient reduced models for multi-body dynamics simulations of helicopter wing missile configuaratons Mechanical Engineering Master Thesis, university of Massachusetts Lowell, 2006.

[38] R. Brincker, C. Ventura, Introduction to Operational Modal Analysis, Wiley, 2015.

[39] LMS Test.Lab 10A, LMS Test.Lab 10A, Leuven Measurement Systems, Leuven, Belgium.

[40] B. Peeters, H. Van der Auweraer, F. Vanhollebeke, P. Guillaume, Operational modal analysis for estimating the dynamic properties of a stadium structure during a football game, Shock and Vibration, Volume 14, Issue 4, 2007, pp. 283-303.

[41] B. Peeters, H. Van der Auweraer, P. Guillaume, J. Leuridan, The PolyMAX Frequency-Domain Method: A New Standard for Modal Parameter Estimation?, Shock and Vibration, Volume 11, Issue 3-4, 2004, doi: $10.1155 / 2004 / 523692$.

[42] J. Baqersad, J. Carr, T. Lundstrom, C. Niezrecki, P. Avitabile, M. Slattery, Dynamic characteristics of a wind turbine blade using 3D digital image correlation, SPIE Symposium on Smart Structures \& Materials/NDE and Health Monitoring, San Diego, CA, April 2012. doi:10.1117/12.915377.

[43] J. Carr, J. Baqersad, C. Niezrecki, P. Avitabile, Full-Field Dynamic Strain on Wind Turbine Blade Using Digital Image Correlation Techniques and Limited Sets of Measured Data From Photogrammetric Targets, Experimental Techniques, 2015, doi: 10.1111/ext.12129. 
[44] J. Carr, J. Baqersad, C. Niezrecki, P. Avitabile, M. Slattery, Dynamic stress-strain on turbine blade using digital image correlation techniques Part 1: Static load and calibration, Jacksonville, FL, February 2012, pp. 215-220. doi:10.1007/978-1-4614-2422-2_20. 